\title{
Activités antibactériennes de l'extrait des feuilles de Dalechampia clematidifolia (Euphorbiaceae)
}

\author{
Lalaina H. TSIRINIRINDRAVO* et Blandine ANDRIANARISOA \\ Département de Biochimie Fondamentale et Appliquée, Faculté des Sciences d'Antananarivo, \\ Université de Madagascar, BP 906, Antananarivo 101, Madagascar. \\ *Auteur correspondant, E-mail: tehachelone@yahoo.fr
}

\section{RESUME}

Dans l'optique de rechercher les antibiotiques d'origine naturelle, l'activité antibactérienne des extraits de feuilles de Dalechampia clematidifolia sur certaines bactéries a été étudiée. Dalechampia clematidifolia est une euphorbe endémique de Madagascar ayant de nombreuses vertus. Elle est utilisée en pharmacopée traditionnelle pour traiter les toux, les maux de ventre, toutes infections de la voie respiratoire, la grippe et les suppurations diverses. Ainsi, compte tenu de l'importance biologique et médicale de cette plante et dans le but de sa valorisation, une étude a été menée. Une extraction aqueuse à froid des feuilles nous a permis d'avoir un extrait brut opaque marron foncé, de $\mathrm{pH} 5,98$ et ayant une concentration de 62,39 $\mu \mathrm{g} / \mu 1$. Des tests d'antibiogramme sur différentes souches bactériennes ont montré que c'est un extrait à large spectre. Cette activité est surtout remarquée vis-à-vis de quelques espèces appartenant à la famille des Enterobactériacées (Shigella boydii, Escherichia coli, Alkalescens dispar, Hafnia alvei et Enterobacter cloaceae), des Micrococacées (Micrococcus luteus, Staphylococcus aureus). La sensibilité de Rhodococcus sp., Listeria monocytogenes, Listeria utelshi, Listeria ivanivii a été également remarquée. Les CMI de cet extrait vis-à-vis de Hafnia alvei, Micrococcus luteus, Staphylococcus aureus, et Listeria utelshi sont respectivement de 15,92 $\mu \mathrm{g} / \mu \mathrm{l}, 22,15 \mu \mathrm{g} / \mu \mathrm{l}, 15,92 \mu \mathrm{g} / \mu \mathrm{l}$, et 18,50 $\mu \mathrm{g} / \mu \mathrm{l}$. Sa CMB vis-à-vis de Hafnia alvei et de Staphylococcus aureus est égale à $48 \mu \mathrm{g} / \mu \mathrm{l}$. Cependant, cet extrait n'a présenté aucune activité sur les espèces de la famille des Vibrionacées. Son utilisation de façon empirique pour traiter différents maux a été donc justifiée par ces activités biologiques.

(C) 2009 International Formulae Group. All rights reserved

Mots-clés: Dalechampia clematidifolia, euphorbe endémique, antibiogramme, larges spectres, CMI, CMB.

\section{INTRODUCTION}

Madagascar est un pays réputé pour sa diversité floristique. De nombreuses plantes sont utilisées pour guérir différents maux (Courvalin, 1992; Tsirinirindravo et al., 2003 ; Tsirinirindravo et al., 2006). D'après nos enquêtes ethnobotaniques, il a été constaté que, l'espèce Dalechampia clematidifolia, connue sous le nom vernaculaire de Teloravina, est utilisée pour soigner efficacement les plaies et pour traiter la grippe, les toux, les maux de gorge, toutes infections de la voie respiratoire. Une activité antibactérienne de cette plante pourrait donc être envisagée. En outre, c'est une espèce endémique de Madagascar, et aucune donnée, autre que botanique n'existe jusqu'à présent sur cette plante.

Compte tenu de l'importance biologique et médicale de cette espèce et dans le but de sa valorisation, une étude scientifique beaucoup plus approfondie a été menée sur cette plante en vue de la découverte d'un nouveau produit antimicrobien (antibiotique, antiseptique) d'origine végétale. Dalechampia clematidifolia est une liane de 2 à $3 \mathrm{~m}$ de long. Les feuilles sont alternes. Le limbe est divisé en 3 folioles relativement larges. Les bractées de couleur blanches sont plus longues que larges, à lobes aigus, 
denticulés, à base ovale, n'atteignant pas 3 $\mathrm{cm}$. Les fleurs sont petites, à sépales libres. L'ovaire est supère, et triloculaire. Il y a un ovule par loge (Mabberley, 1987; Smith, 1987).

\section{MATERIEL ET METHODES \\ Le matériel végétal}

La plante étudiée a été récoltée au pied de l'Ankaratra, dans la région de Vakinankaratra au mois de septembre 2004.

Les feuilles ont fait l'objet de cette étude. Elles ont été lavées délicatement afin d'enlever divers contaminants, puis mises au congélateur.

\section{Les souches bactériennes}

Les espèces bactériennes utilisées sont constituées par des souches typées (ATCC) produites par Microbiologics ainsi que d'autres espèces bactériennes isolées et purifiées, à partir d'inoculum provenant soit des denrées alimentaires, soit d'échantillon de sol, ou de prélèvement pathologique, de frottis cervical.

\section{Extraction}

Les feuilles décongelées sont broyées puis délayées dans de l'eau distillée suivant un rapport $1 / 8(\mathrm{p} / \mathrm{v})$. Le mélange, soumis à une agitation magnétique pendant $3 \mathrm{~h}$ à la température ambiante, est ensuite laissé macérer pendant une nuit à $+4{ }^{\circ} \mathrm{C}$ (Hamon et al., 1998; Bruneton, 1999). Le macérât est de nouveau agité pendant $30 \mathrm{~min}$ à la température ambiante puis filtré avec du papier filtre pour éliminer les tourteaux. Le filtrat obtenu est centrifugé à $16000 x g$ pendant $30 \mathrm{~min}$ à l'aide d'une centrifugeuse réfrigérée SIGMA 3K30 (rotor angulaire).

Le culot est éliminé tandis que le surnageant est concentré sous pression réduite à $55{ }^{\circ} \mathrm{C}$ à l'aide d'un évaporateur rotatif (Heidolph modèle laboratora 4000 efficient) relié à une pompe à vide (Heidolph modèle rotavac).

Une deuxième centrifugation à 16000xg pendant 15 min est indispensable afin d'éliminer le précipité apparu lors de la concentration. L'extrait est constitué par le surnageant.

\section{Tests biologiques}

L'activité antibactérienne de cet extrait est ensuite étudiée sur 21 souches bactériennes, selon la méthode de diffusion sur gélose (Duval, 1990). Ce test se déroule en 3 jours successifs :

Premier jour: chaque souche pure est ensemencée sur bouillon nutritif, puis incubée à $37{ }^{\circ} \mathrm{C}$ pendant $24 \mathrm{~h}$; c'est le relancement de la culture.

Deuxième jour: chaque souche préalablement relancée est diluée de façon à avoir une concentration bactérienne égale à 103 cellules $/ \mathrm{ml}$ : cette préparation constitue l'inoculum (Jorgensen, 1998). Après, 1 ml de cet inoculum est ensemencé uniformément selon la technique d'inondation dans une boîte de Pétri contenant le milieu Mueller Hinton de 4 mm d'épaisseur (Mueller, 1941 ; Jupeau, 1990). Cependant, pour le cas de Streptococcus pneumoniae ATCC 6301, le milieu Mueller Hinton additionné de $5 \%$ de sang de mouton défibriné doit être utilisé (Jorgensen, 1999). La boite est laissée à la température ambiante pendant $1 \mathrm{~min}$ afin que les germes puissent bien adhérer à la surface de la gélose (Larpent, 1997). La boite est séchée à l'étuve à $37{ }^{\circ} \mathrm{C}$ pendant $15 \mathrm{~min}$. Les disques stériles pour antibiogramme (préalablement autoclavés) ont été imprégnés de $20 \mu \mathrm{l}$ d'extrait. Ils sont ensuite déposés à la surface du milieu ensemencé, à l'aide d'une pince fine. La préparation est incubée à $37^{\circ} \mathrm{C}$ pendant $24 \mathrm{~h}$. Chaque test est effectué en 2 exemplaires (Larpent, 1970 ; Meyer , 1994).

Troisième jour: après incubation, le diamètre du halo d'inhibition formé autour de chaque disque est mesuré et leurs moyennes calculées.

Les normes utilisées dans l'expression des résultats des tests de diffusion sur gélose sont données dans le tableau 1. Il s'agit des normes données par le comité de l'antibiogramme de la Société Française de Microbiologie (CA-SFM) en 2006, se basant sur les recommandations du Comité d'Experts de la Standardisation biologique de l'O.M.S en 1977. Leurs CMI étaient ensuite déterminées. Ainsi, après un relancement de chaque souche bactérienne sur du bouillon nutritif à $37{ }^{\circ} \mathrm{C}$ pendant $24 \mathrm{~h}$, chaque culture est diluée de façon à avoir une concentration égale à 103 cellules par ml (Meyer , 1994). Le milieu inoculé est distribué dans 9 tubes à 
Tableau 1: Norme utilisée pour la lecture des résultats des tests d'antibiogramme.

\begin{tabular}{lll}
\hline Diamètre du halo d'inhibition $\Delta$ & Degré de sensibilité du germe & Symbole \\
\hline$\Delta<7 \mathrm{~mm}$ & Insensible & - \\
$7 \mathrm{~mm} \leq \Delta<8 \mathrm{~mm}$ & Assez sensible & + \\
$8 \mathrm{~mm} \leq \Delta<9 \mathrm{~mm}$ & Sensible & ++ \\
$\Delta \geq 9 \mathrm{~mm}$ & Très sensible & +++ \\
\hline (Courvalin, 1992; OMS., 2002; Sinot, 1985). & &
\end{tabular}

hémolyse à raison de $900 \mu \mathrm{l}$ par tube. Parallèlement, une gamme de 9 concentrations d'extrait est préparée selon une raison géométrique égale à 0,5 . Ensuite, $100 \mu \mathrm{l}$ de chacune de ces 9 concentrations, sont additionnés dans chaque tube. Un milieu non inoculé stérile sert de témoin positif (pour la présence d'activité antibactérienne) et un milieu inoculé mais dépourvu d'extrait sert de témoin négatif (Murray, 1995).

Après incubation des tubes à $37{ }^{\circ} \mathrm{C}$ pendant $24 \mathrm{~h}$, la turbidité des tubes traduisant la croissance bactérienne, est notée. La CMI correspond à la plus faible concentration où aucune croissance bactérienne n'est observée après comparaison au témoin positif : milieu limpide (Jorgensen, 1998).

La numération sur milieu solide des cellules viables provenant des cultures en milieu liquide lors de la détermination de la CMI permet d'évaluer la CMB. Pour cela, une oëse calibrée à $10 \mu$ l est ensemencée sur l'agar de Mueller Hinton. Après incubation à $37^{\circ} \mathrm{C}$ pendant $24 \mathrm{~h}$, les boîtes sont observées (Duval, 1990).

La CMB correspond à la concentration minimale pour laquelle toutes les bactéries sont mortes et aucune colonie caractéristique n'est observée sur le milieu (Bechtle, 1958 ; Duval, 1990).

\section{RESULTATS \\ Extraction}

Ce procédé d'extraction a permis d'obtenir un extrait brut opaque, de couleur marron foncé, de $\mathrm{pH}$ égale à 5,6 et ayant une concentration égale à $62,39 \mu \mathrm{g} / \mu 1$.

\section{Tests biologiques}

Les diamètres des halos d'inhibitions de l'extrait brut issu de Dalechampia clematidifolia sur les différents microorganismes sont donnés dans le tableau 2.
D'après ces résultats, de nombreuses espèces bactériennes pathogènes pour l'homme sont sensibles à cet extrait. Cette sensibilité est surtout remarquée pour les espèces de la famille des Enterobactériacées telles que : Shigella boydii, Escherichia coli, Alkalescens dispar, Hafnia alvei et Enterobacter cloaceae.

Parmi les Micrococacées ; Micrococcus luteus et Staphylococcus aureus sont les plus sensibles.

Cet extrait n'est pas actif contre les espèces de la famille des Vibrionacées. La sensibilité de Rhodococcus sp., Listeria monocytogenes, Listeria utelshi, Listeria ivanivii a été également remarquée.

Parmi toutes ces espèces bactériennes, c'est Rhodococcus sp. qui est la plus sensible avec un halo de $18 \mathrm{~mm}$ de diamètre.

Les CMI de cet extrait vis-à-vis de Rhodococcus sp, Hafnia alvei, Micrococcus luteus, Staphylococcus aureus, et Listeria utelshi sont respectivement de 13,40 $\mu \mathrm{g} / \mu \mathrm{l}$, $15,92 \mu \mathrm{g} / \mu \mathrm{l}, 22,15 \mu \mathrm{g} / \mu \mathrm{l}, 15,92 \mu \mathrm{g} / \mu \mathrm{l}$, et $18,50 \mu \mathrm{g} / \mu \mathrm{l}$. Les autres valeurs de CMI correspondant aux autres germes sont supérieures à la concentration initiale de l'extrait. Les CMB de cet extrait vis-à-vis de Hafnia alvei, de Rhodococcus sp et de Staphylococcus aureus sont égales à $48 \mu \mathrm{g} / \mu \mathrm{l}$.

\section{DISCUSSION}

Cet extrait est un produit dit à larges spectres. En effet il est actif vis-à-vis de nombreuses espèces bactériennes, surtout visà-vis de quelques espèces appartenant à la famille des enterobactériacées. Ils peuvent être utilisés pour traiter les maux de ventre, la diarrhée, la dysenterie, bref, les cas de toxiinfection ou d'infections à Enterobactéries. En outre, son activité vis-à-vis des 3 espèces de Listeria (Listeria monocytogenes, Listeria utelshi, Listeria ivanivii) a été signalée. Ils 
Tableau 2: Diamètres des halos d'inhibition de l'extrait issu de Dalechampia clematidifolia.

\begin{tabular}{lclc}
\hline Germes & $\Delta$ & Germes & $\Delta$ \\
\hline Shigella boydii & 15 & Streptococcus faecalis ATCC 19433 & 6 \\
Escherichia coli ATCC 25922 & 9 & Vibrio cholerae ATCC 15748 & 7 \\
Serratia marcescens & 6 & Vibrio parahaemolyticus & 7 \\
Salmonella typhi & 6 & Bacillus cereus & 7 \\
Alkalescens dispar & 10 & Pseudomonas aeruginosa ATCC 9027 & 12 \\
Hafnia alvei & 17 & Rhodococcus sp. & 18 \\
Enterobacter cloaceae ATCC 700323 & 12 & Listeria monocytogenes & 17 \\
Streptococcus pneumoniae ATCC 6301 & 6 & Listeria utelshi & 14 \\
Micrococcus luteus & 14 & Listeria ivanivii & 16 \\
Staphylococcus aureus & 14,5 & Acinetobacter gerneri & 12 \\
\hline
\end{tabular}

pourraient donc être actifs pour traiter la listériose.

Par ailleurs, une activité a été constatée vis-à-vis de Rhodococcus sp. C'est ce qui pourrait expliquer son utilisation par les tardipraticiens pour traiter la grippe. En effet, l'état fébrile d'une personne grippée est dû aux bactéries dites opportunistes entre autres, Rhodococcus sp. (Duval, 1990).

Par rapport aux extraits issus de la plante codée THL de la famille des Astéracées, l'extrait issu de Dalechampia clematidifolia est très performant, de par son large spectre, et également par ses activités bactéricides. En effet, vis-à-vis de Staphylococcus aureus, la CMB de l'extrait issu de la plante codée THL est de 99,75 $\mu \mathrm{g} / \mu \mathrm{l}$ (Tsirinirindravo et al., 2003).

Cet extrait est également très performant par rapport à l'extrait issu de la plante Uapaca thouarsii, une espèce de la famille des Euphorbiacées, surtout de par son spectre très large, et sa faible CMB vis-à-vis de Staphylococcus aureus. La CMB de l'extrait issu de Uapaca thouarsii $(\mathrm{CMB}=131$ $\mathrm{mg} / \mathrm{ml}$ ) est largement supérieure à celle de l'extrait issu de Dalechampia clematidifolia $(\mathrm{CMB}=48 \mu \mathrm{g} / \mu \mathrm{l})$ (Rakotonandrasana, 2004).

\section{REFERENCES BIBLIOGRAPHIQUES}

Bechtle M, Scher R. 1958. Antibiotics and chemotherapy. Proc. Soc. Exp. Path., 59: 559-606.

Bruneton J. 1999. Plantes Toxiques: Végétaux Dangereux pour l'Homme et les Animaux. Tec et Doc Lavoisier: Londres, Paris, New York ; 529p.
Courvalin P. 1992. Interpretative Reading of Antimicrobial Susceptibility Test. ASM Press, 58: 368-375.

Duval SCJ. 1990. Antibiothérapie, (4 édn). Masson : Paris ; 188p.

Hamon M, Mahuzier G. 1990. Abrégé de Chimie Analytique (2éd). Masson: Paris, Milan, Barcelone, Mexico, 2; 259p.

Jorgensen JH, Ferraro MJ. 1998. Antimicrobial susceptibility testing: general principles and contemporary practices. Clin. Infect. Dis., 25: 973-980.

Jorgensen JH, Ferraro MJ. 1999. Antimicrobial susceptibility test: dilution and disc diffusion methods. In Manual of Clinical Microbiology (7 édn), Murray PR, Baron EJ, Pfaller M, Tenover FC, Vulken RH (eds). ASM Press: Washington DC; 1526- 1547.

Jupeau VA, Scavizzi M. 1990. Sensibilité des bactéries aux antibiotiques: méthodes d'études en biologie clinique. In Encyclopédie Médicale Chirurgicale: Maladies Infectieuses. Édition Technique : Paris; 1099p.

Larpent J-P, Larpent-Gourgaud M. 1970. Microbiologie Pratique. Hermann: Paris ; 203p.

Larpent J-P, Larpent-Gourgaud M. 1997. Mémento Technique de Microbiologie (3 édn). Lavoisier: Londres, New York, Paris; 1039 p.

Meyer A, Deiana J, Leclerc H. 1994. Cours de Microbiologie Générale Nouveau Programme. Doin Editeurs : Paris; 495p.

Mabberley DJ. 1987. The Plant-book. Cambridge University press; Cambridge; 706 . 
Mueller JH, Hinton J. 1941. Milieux de cultures. Proc. Soc. Exp. Path., 29: 181183.

Murray, Barron. 1995. Antibacterial susceptibility tests: dilution and disks diffusion. In Manual of Clinical Microbiology, WAP (ed). ASM Press: Washington DC; 1327-1338.

OMS. 2002. L'utilisation des antimicrobiens en dehors de la medecine humaine et les résistances qui en résultent chez l'homme. OMS Aide-Mémoire N²68, Genève.

Rakotonandrasana J. 2004. Etude chimique et toxicologique des extraits toxiques d'écorces de tige de Uapaca thouarsii (Euphorbiaceae). Mémoire de DEA. Université d'Antananarivo, Antananarivo.

Rakotonirainy M, Raison MA. 1999. Activité antifongique de quelques huiles essentielles et produits apparents. In: Les documents graphiques et photographiques: analyse et conservation. Travaux du Centre de Recherche sur la Conservation des documents graphiques 1994-1998. La documentation Française, Paris, pp. 173192.

Sinot J. 1985. Evaluation de l'activité des antibiotiques in vitro. In Bactériologie Médicale, Le MINOR L (ed). Doin Editeur, 343p.

Smith AR. 1987. Flora of Tropical East Africa. Part I: Euphorbiaceae. AA Balkema: Rotterdam, Boston ; 407p.

Tsirinirindravo HL, Andrianarisoa B. 2003. Etude des principes antibactériens d'une plante de Madagascar. Communication à la SFM, Bordeaux.

Tsirinirindravo HL, Andrianarisoa B. 2003. Contribution à l'étude des propriétés antibactériennes de la plante codée THL (Asteraceae). MESRES Forum (Antananarivo, 09-10-11 décembre).

Tsirinirindravo HL, Andrianarisoa B. 2005. Lutte contre les vecteurs du paludisme: place aux insecticides naturels issues de plantes. Communication et manifestation lors de la Vitrine de Madagascar du 27 au 29 octobre 2005. 